\title{
Graphene materials for lead (II) extraction: an equilibrium study
}

\author{
Anastasia Kucherova ${ }^{1, *}$, Irina Burakova ${ }^{1}$, and Alexandr Burakov ${ }^{1}$ \\ ${ }^{1}$ Tambov State Technical University, Department “Technology and Methods of Nanoproducts Manufacturing”, 392000, 106 Sovetskaya \\ Str., Tambov, Russia
}

\begin{abstract}
The present paper describes a study on the adsorption of lead (II) ions on graphene materials obtained via exfoliation of a hydrolyzed expanded graphite intercalation compound. An equilibrium studies were performed implementing the empirical Freundlich and Langmuir isotherm models. It was found that all the isotherms are fitted well by those models, indicating the efficiency of the graphene materials as an adsorbent of heavy metals. The applicability of the two isotherms for sorption processes shows that both monolayer adsorption and heterogeneous energetic distribution of active sites on the adsorbent surface may take place. The maximum experimental lead (II) adsorption capacities of the materials under study - fewlayered oxidized graphene suspension, multi-layered graphene paste, and few-layered graphene paste - were found to be 850,230 and $170 \mathrm{mg} / \mathrm{g}$, respectively. Due to the high sorption characteristics and unique physical and chemical properties of these materials, the adsorption technologies developed herein can act as good sustainable options for the future in heavy metal removal from industrial effluents.
\end{abstract}

\section{Introduction}

Rapid industrialization of human society leads to the fact that different contaminants (such as heavy metal ions, organics, bacteria, viruses, etc), which are seriously harmful to human health, are released to wastewater. Among all the water pollutants, heavy metal ions, including $\mathrm{Pb}(\mathrm{II}), \mathrm{Cd}(\mathrm{II}), \mathrm{Zn}(\mathrm{II}), \mathrm{Ni}(\mathrm{II})$ and $\mathrm{Hg}(\mathrm{II})$, have high toxicity and non-biodegradable properties and can cause severe health problems in animals and human beings. For instance, lead poses several risks to human health, causing insomnia, pain, dizziness, anemia, irritability, muscle weakness, hallucinations, and renal damage [1].

The presence of the above-mentioned heavy metals in aqueous systems poses a serious environmental problem. In this regard, various techniques, such as ion exchange, membrane filtration, coagulation, flocculation, flotation and electrochemical methods, have been proposed to remove and isolate those species [2]. Among them, adsorption appears to be the most efficient way. It has been widely employed in separation, waste effluents treatment, refrigeration, environmental control, life support devices in spacecraft, and heterogeneous catalysis [3-5].

Graphene, a new carbon nanomaterial, possesses unique physical, chemical, electrical and mechanical properties [6-9], and is adequate for removing heavy metals from aqueous solutions. It has demonstrated high adsorption rates and fast adsorption kinetics [10-13].

Considering the aforementioned, the aim of the present research was to develop novel graphene materials for efficiently extracting $\mathrm{Pb}(\mathrm{II})$ from aqueous solutions.
Electron images of graphene-based samples were obtained by scanning electron microscopy (SEM) on a Neon 40 two-beam instrument (Carl Zeiss, Jena, Germany) and transmission electron microscopy (TEM) on a JeM-200CX instrument (JEOL, Tokyo, Japan). The equilibrium $\mathrm{Pb}$ (II) concentration in aqueous solutions was determined by atomic absorption spectrometry (AAS) on an MGA-915MD instrument (Atompribor Ltd., Saint-Petersburg, Russia).

\section{Experimental part}

\subsection{Adsorbents}

1) Graphene samples under study represent aqueous pastes containing multi- or few-layered graphene.

The graphene pastes were synthesized according to the method described in [14]. Graphite was intercalated with an ammonium persulfate solution in sulfuric acid, and then aged for $3 \mathrm{~h}$ at $40{ }^{\circ} \mathrm{C}$ for expansion. After that, it was hydrolyzed and washed with water until the acid was removed. The addition of surfactants promotes the formation of few-layered graphene. As a rule, the graphene content in the multi-layered graphene paste (MLGP) was 5 wt.\%, and in the few-layered graphene paste - 12 wt.\% (based on graphene carbon). The graphene contained about 10 wt.\% of oxide groups related to the graphene carbon.

The typical SEM and TEM images of the MLGP are presented in Fig. 1, from which it can be seen that the size of nanoplatelets is on the order of 5-10 nm, and the number of layers is $15-25$.

\footnotetext{
Corresponding author: anastasia.90k@mail.ru
} 
As seen in Fig. 2, the FLGP represents few-layered graphene nanoplatelets with the size of about $5-10 \mathrm{~nm}$ and the number of layers of 3-5.

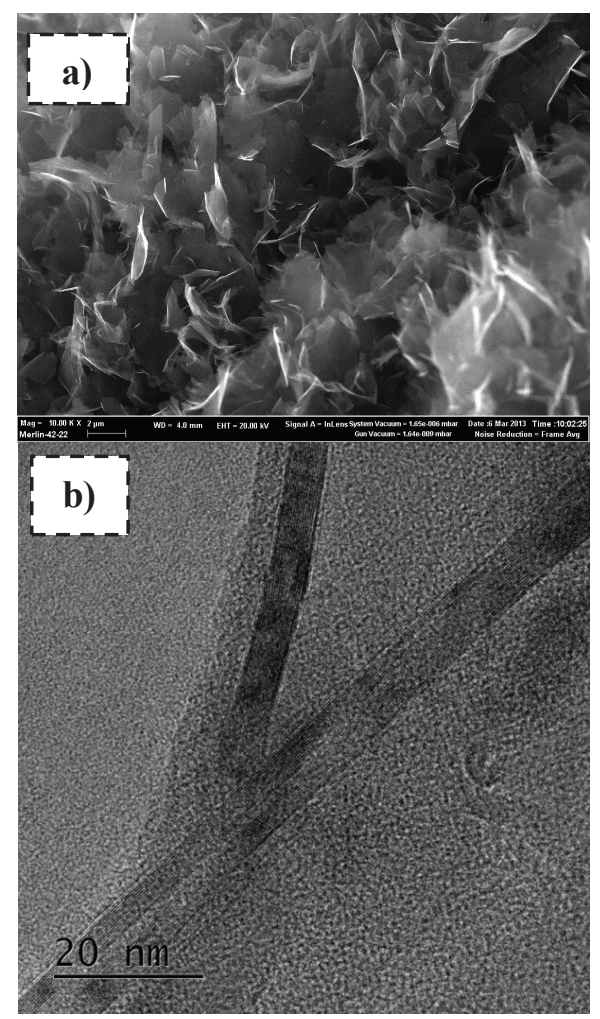

Fig. 1. SEM (a) and TEM (b) images of the MLGP.

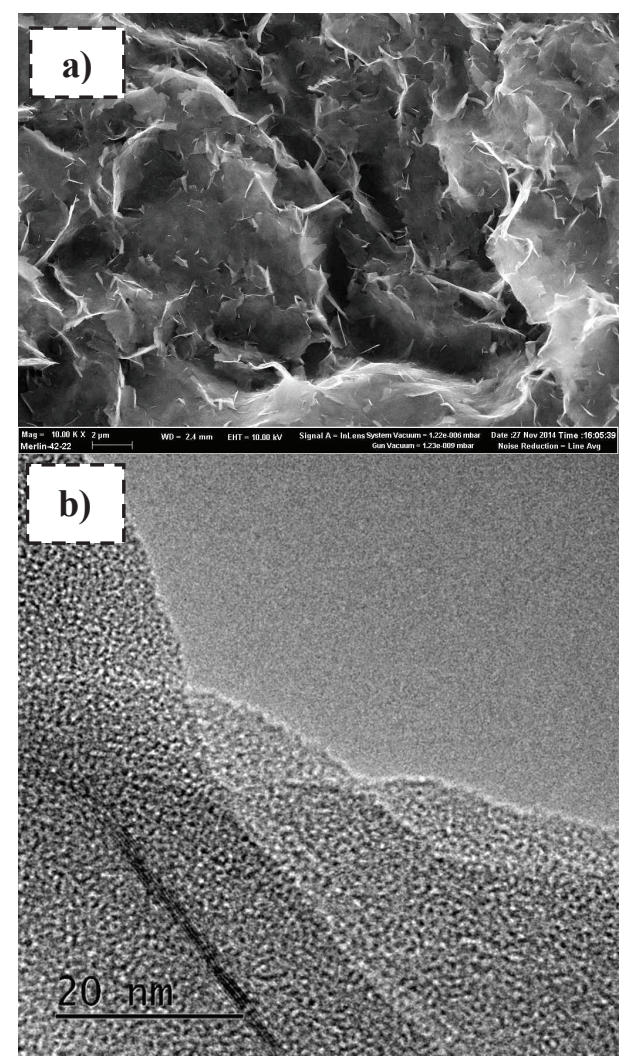

Fig. 2. SEM (a) and TEM (b) images of the FLGP.

2) Few-layered oxidized graphene suspension (FLOGS) represents an aqueous dispersion of oxidized few-layered graphene nanoplatelets. This dispersion was prepared by forming an expanded graphite compound according to the procedure described elsewhere [15], and then stabilized using a Fenotam GR-326 water-soluble phenol-formaldehyde resole resin (PFR) (Pigment Ltd., Tambov, Russia) containing $50 \%$ of non-volatile substances. GSM-2 natural graphite (Pavna Ltd., Belgorod, Russia), containing $\leq 0.5 \%$ of mineral impurities, was intercalated by ammonium persulfate in anhydrous sulfuric acid monohydrate (both - reagent grade, Laverna Ltd., Moscow, Russia). After that, the intercalated compound was expanded for $3 \mathrm{~h}$ at $40^{\circ} \mathrm{C}$, and then filtered and washed with water to neutral $\mathrm{pH}$. The obtained hydrolyzed expanded graphite compound was used for ultrasonic exfoliation without drying. The calculated amount of wet HEGC, obtained after mixing 5 $\mathrm{g}$ of the starting graphite and $5 \mathrm{~g}$ of the PFR, was placed into a 2-L beaker. Next, water was added to this mixture until a total mass of $1,500 \mathrm{~g}$ was obtained. The resulting suspension was sonicated using an IL10 ultrasonic generator (Ultrasonic Technique-INLAB Ltd., SaintPetersburg, Russia) under continuous stirring for $6 \mathrm{~h}$. The graphene content in the obtained colloidal dispersion was $3.33 \mathrm{~g} \mathrm{~L}^{-1}$, and the PFR content (on a dry basis) was $1.67 \mathrm{~g} \mathrm{~L}^{-1}$.

\subsection{Research methodology}

The experimental study on the $\mathrm{Pb}(\mathrm{II})$ adsorption from aqueous solutions was performed with the FLOGS, FLGP and MLGP graphene materials used as adsorbents. Each series consisted of seven $50-\mathrm{mL}$ tubes containing the corresponding adsorbent. $15 \mathrm{~mL}$ of $\mathrm{Pb}\left(\mathrm{NO}_{3}\right)_{2}$ solutions having concentrations of 200,500, 700, 1,000, 2,400 and $3,500 \mathrm{mg} / \mathrm{L}$ were separately added into those tubes using Pasteur pipettes. Then, the tubes were endover-end shaken on Bio RS-24 programmable rotators (Biosan, Riga, Latvia) at $100 \mathrm{rpm}$ during the equilibrium time previously found for the adsorbent. After the adsorption process was finished, the solutions were centrifuged using a $5810 \mathrm{R}$ centrifuge (Eppendorf, Hamburg, Germany) at $10,000 \mathrm{rpm}$ for $10 \mathrm{~min}$ to separate the phases. Next, the liquid phase was placed into $30-\mathrm{mL}$ tubes with the Pasteur pipettes, preventing ingress of the solid phase and the suspension thereof. Finally, the solutions were measured according to the method of quantitative elemental analysis by atomic absorption spectra on a spectrometer.

The equilibrium $\mathrm{Pb}(\mathrm{II})$ adsorption capacity, $\mathrm{q}_{\mathrm{e}}$ $(\mathrm{mg} / \mathrm{g})$, was evaluated according to Eq. (1):

$$
q_{e}=\left(C_{0}-C_{e}\right) \cdot V / m
$$

where $\mathrm{V}$ is the sample volume (L), $\mathrm{m}$ is the mass of the adsorbents $(\mathrm{g}), \mathrm{C}_{\mathrm{o}}$ is the initial $\mathrm{Pb}(\mathrm{II})$ concentration $(\mathrm{mg} / \mathrm{L})$, and $\mathrm{C}_{\mathrm{e}}$ is the equilibrium $\mathrm{Pb}(\mathrm{II})$ concentration in the solution $(\mathrm{mg} / \mathrm{L})$. 


\section{Results and discussion}

To optimize the design of an adsorption system for adsorbate removal, it is important to establish the most appropriate correlation for equilibrium data. Two adsorption isotherm models - Langmuir and Freundlich were implemented herein to fit the experimental data. The Langmuir model assumes that the adsorption takes place at specific homogeneous sites located on the adsorbent surface. Its form is given in Eq. (2) as follows:

$$
q_{e}=\frac{q_{\max } b C_{e}}{b C_{e}+1}
$$

where, $q_{e}$ is the $\mathrm{Pb}(\mathrm{II})$ amount adsorbed at equilibrium per unit weight of adsorbent $(\mathrm{mg} / \mathrm{g}), q_{\max }$ is the saturated monolayer adsorption capacity $(\mathrm{mg} / \mathrm{g}), b$ is the Langmuir constant related to the binding energy $(\mathrm{L} / \mathrm{mg})$ of the adsorption system, and $C_{e}$ is the $\mathrm{Pb}$ (II) equilibrium concentration $(\mathrm{mg} / \mathrm{L})$.

The Freundlich model can be applied to non-ideal sorption onto heterogeneous surfaces in multiple layers. It is expressed by Eq. (3) as follows:

$$
q_{e}=k_{f}\left(C_{e}\right)^{1 / n}
$$

where $q_{e}$ is the $\mathrm{Pb}(\mathrm{II})$ amount adsorbed at equilibrium $(\mathrm{mg} / \mathrm{g}), C_{e}$ is the $\mathrm{Pb}(\mathrm{II})$ equilibrium concentration $(\mathrm{mg} / \mathrm{L}), k_{f}$ is the equilibrium constant which indicates the adsorption capacity, and $\mathrm{n}$ is the equilibrium constant standing for the adsorption intensity (for $1 / n=1$, the adsorption is linear; $1 / n<1$ implies that the adsorption is of the chemical nature, and if $1 / n>1$, the adsorption is favorable and of the physical nature).

Although many other isotherm models have been developed and employed [16], it can be seen that the Langmuir and Freundlich isotherms still remain the two most commonly used equilibrium adsorption equations due to their simplicity and the ease of estimation of their adjustable parameters.

As seen in Figs. 3 and 4, the isotherms can be well approximated by straight lines, thereby confirming the applicability of both the Langmuir and the Freundlich models for describing the $\mathrm{Pb}$ (II) adsorbtion on all the graphene materials under study.

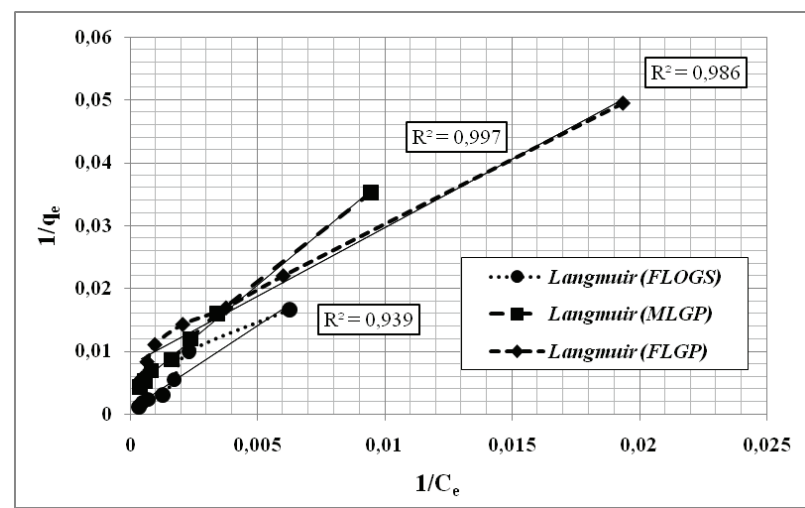

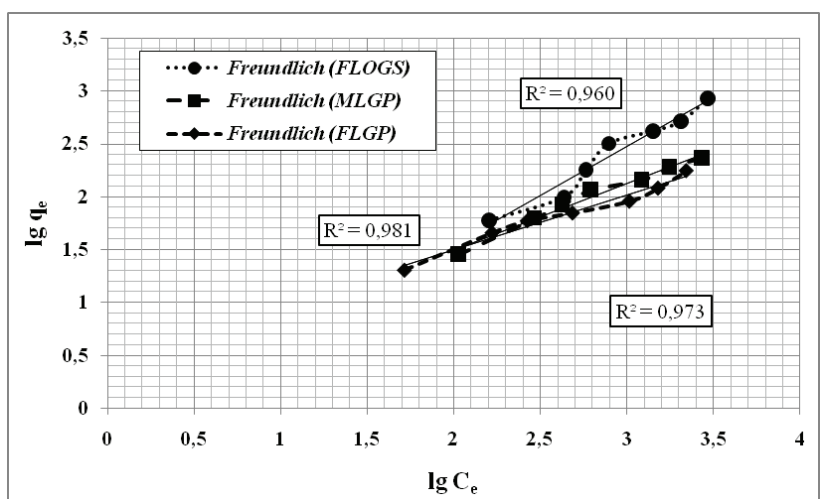

Fig. 4. Freundlich isotherm curves.

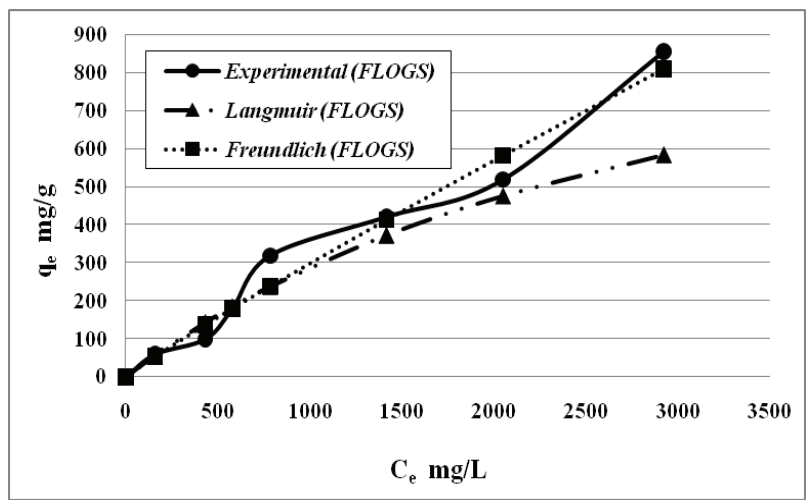

Fig.5. Experimental and theoretical isotherms of the $\mathrm{Pb}(\mathrm{II})$ adsorption on the FLOGS.

According to the classification proposed by Smith, the adsorption isotherms constructed for the FLOGS are reminiscent of Type III S-shaped isotherms (Fig. 5), thereby indicating a transitional porous structure of the adsorbent characterized by complicated topology of the pore space and a large variance in the distribution of pore sizes. Furthermore, the nature of the steepness of that isotherm suggests the presence of predominantly very small pores in the adsorbent. The lower part of the S-shaped curve, from the origin to the inflection point, corresponds to the formation of a monomolecular layer, and then turns into multi-molecular adsorption, thereby explaining the further rise of the curve [17-19].

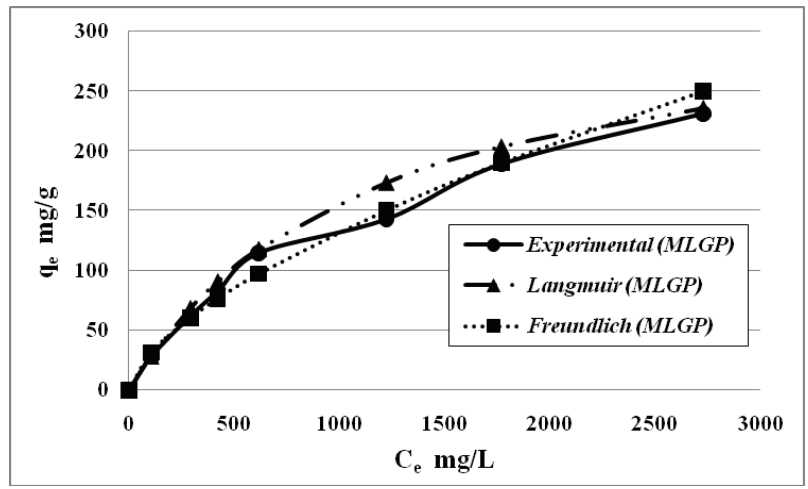

Fig.6. Experimental and theoretical isotherms of the $\mathrm{Pb}$ (II) adsorption on the MLGP.

Fig. 3. Langmuir isotherm curves. 


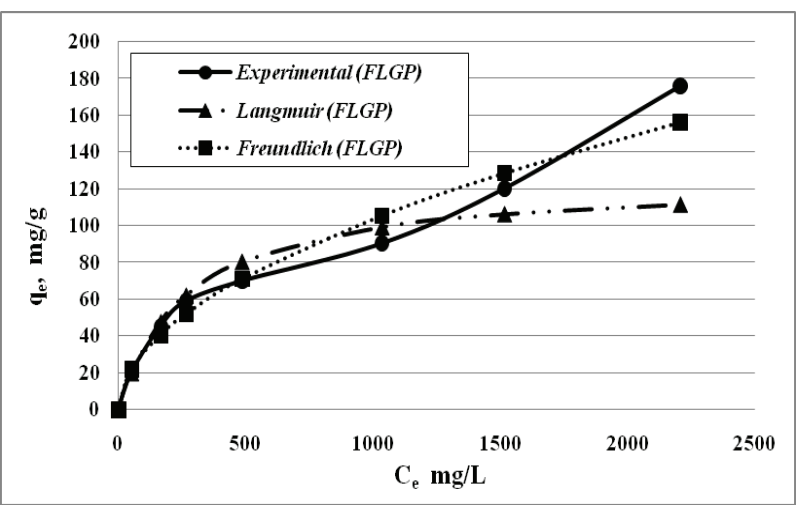

Fig. 7. Experimental and theoretical isotherms of the $\mathrm{Pb}(\mathrm{II})$ adsorption on the FLGP.

After studying the adsorption isotherms constructed for the MLGP and FLGP, it can be assumed that they correspond to L-type curves. In Figs. 6 and 7, an initial curved section can be observed relative to the concentration axis, evidencing the formation of a monomolecular layer. The presence of a second plateau after saturation of the adsorption layer is typical for Type IV isotherms and may be due to multi-molecular adsorption [17].

From the isotherm studies (Table 1), it was found that all the isotherms fit well the experimental data, thereby indicating the efficiency of the graphene materials used herein as heavy metal adsorbents.

It should be noted that the maximum experimental adsorption capacity of the materials under study changes in the following sequence: FLOGS $>$ MLGP $>$ FLGP.

Comparing the correlation coefficients $\left(\mathrm{R}^{2}\right)$ for the two models used, it can be seen that their values are commensurable. This indicates that the $\mathrm{Pb}$ (II) adsorption on the graphene materials has a complex nature, in particular, due to the availability of active sites with different binding energies on the adsorbent surface.

In the range of small concentrations $(200 \ldots 700$ $\mathrm{mg} / \mathrm{L}$ ), it is preferable to implement the Langmuir model. Based on the obtained values of the adsorption equilibrium constants of that equation, it can be assumed that the $\mathrm{Pb}$ (II) ions in their small concentrations interact with active sorption sites located at the surface of the materials under study and responsible for physical adsorption. The range of high concentrations does not satisfy the boundary conditions for the applicability of the Langmuir model with the monomolecular sorption mechanism, and the active adsorption within this range can serve as the foundation for an indirect confirmation of the applicability of the Freundlich model.

The $1 / n$ values obtained can imply favorable conditions for chemical adsorption to occur, since $1 / n<$ 1. Such an adsorption type is related to the energy heterogeneity of adsorption sites.

Based on the systematization of the above-described results, some recommendations on the use of the graphene materials in the $\mathrm{Pb}(\mathrm{II})$ liquid-phase adsorption may be proposed, since the empirical dependencies obtained (Table 2) allow for predicting parameters of the adsorption process.

\section{Conclusions}

The present study demonstrates the metal adsorption capacity of the graphene materials in aqueous solutions. It confirmed the potential of those materials for employing them as efficient adsorbents to extract metal pollutants at both their lower and higher levels in the liquid phase. This finding may be of great significance for industrial wastewater treatment.

Table 1. Isotherm constants obtained for the $\mathrm{Pb}$ (II) adsorption on the FLOGS, MLGP and FLGP materials

\begin{tabular}{l|c|c|c|c|c|c}
\hline \multirow{2}{*}{ Material } & \multicolumn{3}{|c|}{ Langmuir constants } & \multicolumn{3}{c}{ Freundlich constants } \\
\cline { 2 - 7 } & $\boldsymbol{q}_{\max }$ & $\boldsymbol{b}$ & $\boldsymbol{R}^{\mathbf{2}}$ & $\boldsymbol{k}_{\boldsymbol{f}}$ & $\mathbf{1} / \boldsymbol{n}$ & $\boldsymbol{R}^{\mathbf{2}}$ \\
\hline FLOGS & 1250 & $3.01 \cdot 10^{-4}$ & 0.939 & 0.488 & 0.929 & 0.960 \\
\hline MLGP & 333.33 & $8.89 \cdot 10^{-4}$ & 0.997 & 1.659 & 0.634 & 0.981 \\
\hline FLGP & 250 & $3.45 \cdot 10^{-4}$ & 0.989 & 2.850 & 0.520 & 0.908 \\
\hline
\end{tabular}

Table 2. The Langmuir and Freundlich equations obtained based on the experimental data

\begin{tabular}{c|c|c}
\hline \multirow{2}{*}{ Material } & Langmuir & Freundlich \\
\cline { 2 - 3 } FLOGS & $q_{e}=\frac{1250 \cdot 3.01 \cdot 10^{-4} C_{e}}{3.01 \cdot 10^{-4} C_{e}+1}$ & $q_{e}=0.488\left(C_{e}\right)^{0.929}$ \\
\hline MLGP & $q_{e}=\frac{333.33 \cdot 8.89 \cdot 10^{-4} C_{e}}{8.89 \cdot 10^{-4} C_{e}+1}$ & $q_{e}=1.659\left(C_{e}\right)^{0.634}$ \\
\hline FLGP & $q_{e}=\frac{250 \cdot 3.45 \cdot 10^{-4} C_{e}}{3.45 \cdot 10^{-4} C_{e}+1}$ & $q_{e}=2.85\left(C_{e}\right)^{0.52}$ \\
\hline
\end{tabular}


The materials presented high $\mathrm{Pb}(\mathrm{II})$ adsorption rates and large adsorption capacity (up to $850 \mathrm{mg} / \mathrm{g}$ ) under normal conditions. Hence, an extensive research in this area is essential for developing this environmentally friendly process as sustainable technology for the future.

The research was funded by the Ministry of Education and Science of the Russian Federation (Project No. 16.1384.2017/PCh).

\section{References}

1. R. Naseem and S. S. Tahir, Water Res. 35 (2001)

2. F. Fu., Q. Wang, J. Environ. Manage., 92, 3 (2011)

3. Y. Sudhakar, A.K. Dikshit, Int. J. Environ. Poll. 15 (2001)

4. L.R. Radovic, C. Moreno-Catilla, J. Riveral-Utrilla, Chem. Phys. Carbon, 27 (2000)

5. F. Derbyshire, M. Jagtoyen, R. Andrews, A. Rao, I. Martin-Guillon, E.A. Grulke, Chem. Phys. Carbon, 27 (2001)

6. K.S. Novoselov, A.K. Geim, S.V. Morozov, D. Jiang, Y. Zhang, S.V. Dubonos, I.V. Grigorieva, A.A. Firsov, Science, 306 (2004)

7. C.G. Navarro, M. Burghard, K. Kern, Nano Lett., 8 (2008)

8. S. Park, K.S. Lee, G. Bozoklu, W. Cai, S.T. Nguyen, R.S. Ruoff, ACS Nano, 3 (2008)

9. A.A. Balandin, S. Ghosh, W. Bao, I. Calizo, D. Teweldebrhan, F. Miao, C.N. Lau, Nano Lett., 8 (2008)

10. V. Chandra, K.S. Kim, Chem. Commun., 47 (2011)

11. X. Deng, L. Lü, H. Li, F. Luo, J. Hazard. Mater., 183 (2010)

12. S.T. Yang, Y. Chang, H. Wang, G. Liu, S. Chen, Y. Wang, Y. Liu, A. Cao, J. Colloid Interf. Sci., 351 (2010)

13. N. Zhang, H. Qiu, Y. Si, W. Wang, J. Gao, Carbon, 49 (2011)

14. A.V Melezhyk,. A.G. Tkachev, Nanosystems: Physics, Chemistry, Mathematics 5, 2 (2014)

15. A.E. Kucherova, I.V. Romantsova, A.E. Burakov, M.N. Krasnyansky, N.R. Memetov, GrapheneBased Nano-composites for Enhanced $\mathrm{Pb}^{2+}$ Adsorption / NHC, 13 (2016)

16. K.V. Kumar, and S. Sivanesan, J. Hazard. Mater. 136 (2006)

17. G.D. Parfitt and C.H. Rochester. Academic press (1983)

18. T.P. Dyachkova, Yu. A. Khan, N. V. Orlova, S. V. Kondrashov, Vestnik TSTU. 324 (2016)

19. A. G.Tkachev, D. V.Tarov, V. P.Tarov, I. N.Shubin, VestnikTSTU. 503 (2016) 